\title{
La cause caduque des femmes dans l' Égalité des hommes et des femmes de Marie de Gournay
}

\author{
Constant Venesoen \\ The University of Western Ontario
}

La première version de l'Égalité des hommes et des femmes ${ }^{1}$ de Marie de Gournay date de 1622. Oeuvre pleine de verve, sinon de quelque passion agressive, le petit traité écrit en faveur de l'égalité des femmes réservait malgré tout un mérite quelque peu équivoque aux femmes célèbres du passé. En effet, il n'est pas toujours très clair, par exemple, de quel type d'égalité il s'agirait. Égalité devant Dieu? ce qui relève de l'argument passe-partout. Égalité au combat? à quoi l'exemple rabattu des Amazones vient prêter main forte. Égalité des vertus, à la suite de Plutarque qui parlait surtout du courage des femmes? Égalité biologique? puisque Marie de Gournay se permet de rire (et c'est bien la seule et unique fois!) en rappelant plaisamment " $q u$ 'il n'est rien plus semblable au chat sur une fenestre que la chatte" (49).

Le lecteur a cependant le droit de penser que Marie de Gournay songeait surtout à une égalité intellectuelle, et qu'une telle égalité ne relevait finalement que d'une question d'éducation; bref, qu'il suffisait qu'une femme ait reçu une bonne formation intellectuelle pour qu'elle devienne aussitôt et sans la moindre difficulté l'égale de n'importe quel homme.

Les premières pages du traité de 1622 ne manquent pas de souligner cette simple vérité à propos de la "suffisance" des femmes, et des efforts des hommes, "joyeux van- 
teurs," de les "abestir"(41). L'évocation de Diotime et d'Aspasie, voire d'Hypathia, corrobore explicitement le sens de cette égalité de l'esprit. Une allusion à Xénophon qui ne "les regarde selon l'ignorance et l'inexperience où elles sont nourries" (43), évoquant ainsi une condition féminine qui défend souvent aux femmes l'accès au savoir, précise davantage les convictions de Marie de Gournay: "l'institution" des femmes est traditionnellement lacuneuse et leur "nourriture" reste nettement insuffisante. C'est donc bien là que le bât blesse, et que s'offrait à Mademoiselle de Gournay la belle occasion d'écrire la première Histoire de l'égalité intellectuelle des femmes.

Malheureusement Marie de Gournay ne nous propose la plupart du temps qu'une panoplie de femmes célèbres, certes, les unes l'étant un plus que les autres, mais sans que leur "suffisance" soit en cause, voire historiquement attestée. Ce qui est même un peu embarrassant, c'est que de nombreuses femmes évoquées en 1622, et dans les éditions subséquentes, ne se sont pas forcément distinguées par leur haut niveau culturel ou par leur vivacité d'esprit, mais plutôt par leur naissance, leur piété ou leur courage. Marie de Gournay nomme en premier lieu Marie de Médicis et de Catherine de Médicis; puis elle fait une allusion à Procula, femme de Pilate, qui, comme les Sibylles que l'auteur n'oublie pas non plus, a eu le génie de l'intuition, ce dernier bastion de l'intelligence féminine, que tous les hommes concèdent volontiers à leurs compagnes, tout en soupirant. Il est aussi question de Thesbé (sic) [Phoebe dans Paul, Aux Romains, XVI, 1-2], de sainte Pétronille, de Marie-Madeleine, d'Anne, fille de Phanuel. Il y a même une allusion à Marie, mère de Jésus, sur laquelle nous reviendrons. Également à l'appel se présentent la redoutable Judith et l'invincible Pucelle d'Orléans. Parmi les femmes abandonnées, mais pourvues d'un sceptre, trône Didon, tandis que Pentasilée, ${ }^{2}$ mortellement mais héroïquement blessée par Achille quel honneur!, se voit ainsi immortalisée. Mais après tout, nous nous égarons ici en compagnie de Marie de Gournay dans le merveilleux monde des légendes.

Plus crédibles sans doute seraient alors Olda [ou Houlda] et Déborah, ajoutées furtivement dans la marge, comme une arrière-pensée, les deux prophétesses ayant été mentionnées sans fanfare dans la Bible. ${ }^{3}$ Ainsi, dans l'optique de la fameuse "suffisance," il ne resterait finalement que Diotime, dont parle Socrate dans Le Banquet de Platon, Aspasie, compagne illustre de Périclès, ${ }^{4}$ et Hypathia, ${ }^{5}$ cette dernière étant nommée assez timidement dans la marge, alors qu'elle est peut-être la plus savante de toutes. Si Marie de Gournay a cru convaincre tous ces hommes, qu'elle qualifie rageusement de "buffles," de l'égalité intellectuelle des femmes, elle s'y est finalement 
assez maladroitement... attelée. En effet, la majorité des femmes qu'elle mentionne, tout en étant dignes d'admiration pour diverses raisons, ne font pas le poids auprès des doctes.

Il y a plus. C'est que Marie de Gournay n'arrive pas à détacher l'image féminine de la seule supériorité que les misogynes invétérés lui aient jamais reconnue, à savoir son charme et sa beauté. Noémi Hepp a magistralement démontré en quoi consistait alors le "mérite des dames," et à propos de L'Égalité de Marie de Goumay, qu'elle range parmi "les plus féministes des femmes," elle rappelle que la combative demoiselle estimait, elle aussi, que "la beauté" restait le seul apanage de "l'être féminin"6 C'est la raison pour laquelle les femmes devaient, selon saint Paul, garder le silence "en l'eglise," afin qu'elles "n'esmeuvent les tentations" avec leur "grace et beauté" (51). L'explication fait sourire parce qu'elle révèle chez Marie de Gournay une nette perception traditionnelle, oserait-on dire masculine, de la femme séduisante malgré elle. Tout comme lorsqu'elle explique que Jésus se devait d'être un homme, car s'il avait été femme, il n'aurait pas pu "sans scandale (se) mesler jeune et à toutes les heures du jour et de la nuict parmy les presses" (56). Bref, la bienséance féminine, tout comme la modestie, ont empêché Dieu d'avoir une fille! Et pour couronner sa vision médiévale de la femme, la bonne demoiselle rappelle que Jésus fut quand même le fils d'une femme "unique à porter nom de parfaicte entre toutes les creatures purement humaines" (56), cette perfection étant évidemment liée à sa pureté sexuelle, ou, si l'on préfère, à sa virginité immaculée.

En réalité, au lieu d'exalter, preuves à l'appui, la "capacité" féminine mot dont elle orne ses remarques liminaires -, Marie de Gournay fondait sa dialectique sur les seuls témoignages généreux et condescendants d'hommes célèbres: Plutarque, Platon, Aristote, Tacite, saint Jérôme et saint Basile, Montaigne évidemment , pour ne mentionner que les plus illustres. Cette défense collective de la cause indéterminée des femmes sous des plumes souvent suspectes ${ }^{7}$ est loin d'emporter l'adhésion.

Dans la première édition de ses oeuvres complètes, en 1626, sous le titre L'Ombre de la damoiselle de Gournay, l'Égalité réapparaissait. Par un trait qui caractérisera assez bien toutes ses oeuvres quand elles sont réécrites, le texte de 1622 a été modifié ça et là, mais il n'a pas pour autant élargi l'éventail de femmes célébrées pour leur savoir. Le seul nouveau nom est Eustochium, sainte femme et pieuse correspondante virginale de saint Jérôme. ${ }^{8}$ 
En 1634, puis en 1641, Marie de Gournay réédite son oeuvre, cette fois sous le titre de Advis et Presens de la demoiselle de Gournay. A côté de plusieurs changements textuels, dont Mario Schiff a fait un relevé exhaustif, ${ }^{9}$ l'Égalité comporte maintenant un nombre beaucoup plus impressionnant de femmes qui ont acquis quelque renommée au cours de l'Histoire. De toute évidence Marie de Gournay a voulu combler une lacune et consolider sa première dialectique grâce à cet enrichissement quantitatif, voire qualitatif. A ses yeux, n'en doutons pas, la place réservée aux femmes constituerait cette fois un argument massue, sinon inattaquable, en faveur de l'égalité intellectuelle, car n'a-t-on pas "ouy trompetter par les ruës que les femmes manquent de dignité, manquent aussi de suffisance, voire du temperament et des organes pour arriver à cette-cy" (41) (...) et que "la plus haute suffisance à leur advis où les femmes puissent arriver, c'est de ressembler le commun des hommes"? (41) Ce cri de guerre, qui remonte à 1622 , était maintenant devenu un cri de ralliement.

Contrairement à la version de 1622 , celles de 1634 et de 1641 vont nettement miser sur une nomenclature féminine destinée à convaincre les plus sceptiques. Intention louable, certes, mais qui risque cependant d'affaiblir la fougue malgré tout contagieuse du premier jet. En effet, un certain choix d'exemples de femmes, qui n'est pas forcément judicieux, pourrait laisser un arrière-goût de surenchère et, le cas échéant, atteindre un but contraire à celui que s'était proposé l'auteur.

Qui sont elles, ces femmes célèbres qui, selon les prémisses les plus vraisemblables de l'Égalité des hommes et des femmès, sont appelées à prouver l'égalité intellectuelle des sexes? La liste de 1634 est assez longue, et même amplifiée en 1641. Certains noms peuvent convaincre, mais d'autres laissent rêveur et ternissent le prestige des premiers.

Themistocléa (1634 et 1641) est mentionnée par Diogène Laërce, et son copiste Suidas: "Aristoxenus, écrit Diogène, dit que Pythagore reçut la plupart de ses principes moraux de la prêtresse delphique Themistocléa" 10 Éloge de taille, même si le savant Gilles Ménage doute qu'elle fût la soeur de Pythagore. ${ }^{11}$ Quoi qu'il en soit, Marie de Gournay a pris ses sources à la lettre et, conformément à ses intentions, présente donc une femme exemplaire.

Théano, dite la femme de Pythagore, selon Diogène Laërce et Porphyry, est également présente en 1634 et 1641. Mlle de Gournay l'appelle "la sage Theano," se souvenant sans doute avec quelque satisfaction que son correspondant Juste Lipse lui 
avait écrit un jour qu'elle serait la future Théano de son époque. Compte tenu de la gravité de l'humaniste hollandais, on n'oserait imaginer que ce dernier s'était secrètement moqué des moeurs matrimoniales fort libérales de Théano, et a contrario de la rigidité morale de la vieille fille! Quoi qu'il en soit, Théano a certainement fait honneur aux femmes. Stobaeus a dit qu'elle était l'auteur d'un ouvrage de piété. Clément d'Alexandrie et Suidas ont parlé de sa poésie. Lucien a fait l'éloge de sa grandeur d'âme. Enfin, Théodoret a rapporté que Théano avait entrepris de diriger l'école pythagoricienne après la mort de son mari ${ }^{12}$. En fait, cette Théano, tout comme Themistocléa, méritaient plus qu'une simple mention dans l'Égalité de 1634 et de 1641. Mais c'est là une des regrettables omissions de Marie de Gournay: trop fidèle à la méthode de Montaigne, elle ne voit pas la nécessité de justifier ses choix ou de fournir quelques détails explicatifs qui donneraient du poids aux exemples évoqués.

Théano est suivie de Damo, la fille de Pythagore, selon Diogène Laërce. C'est en évoquant l'ento urage familial de Pythagore soeur, femme, fille que Mademoiselle de Gournay la nomme dans un même souffle. Le seul mérite toutefois de Damo, semble-t-il, repose dans son respect filial, puisqu'il est dit qu'elle refusa de livrer les mémoires de son père qui lui avait ordonné de ne les confier à personne. La seule chose que l'on retienne de Damo, c'est donc son obéissance inflexible, ce qui est loin de donner la mesure de son éventuelle stature intellectuelle. Et c'est cette désinvolture dans l'égrènement des noms, marquée par un désir de surenchère, qui risque de miner la thèse dite "féministe" de Marie de Gournay.

L'exemple qui suit est plus heureux. Il s'agit de Cornélia, la mère des Gracques. Marie écrit en 1634 et 1641: "Nous lisons en Ciceron mesme, le prince des orateurs, quel lustre et quelle vogue avoient à Rome et près de luy l'éloquence de Cornelia mere des Gracches: et de plus, celle de Laelia, etc." En effet, on lit dans le Brutus de Cicéron que "Gracchus, par les soins de Cornélia, sa mère, reçut de l'instruction dans son enfance et fut formé aux lettres grecques;" et plus loin: "Nous pouvons lire les lettres de Cornélie, mère des Gracches: il en résulte avec évidence que ses fils ont été formés moins par les tendres attentions que par le pur langage de leur mère."13 Quintilien dit la même chose ${ }^{14}$, et Plutarque, source constante de Marie de Gournay, dit de Cornélia et de ses fils, qu'elle les "nourrit et institua si diligemment, qu'étant devenus plus honnêtes et mieux conditionnés que muls autres jeunes hommes romains de leur temps, on estima que la nourriture en valait mieux que la nature."15 Ces quelques témoignages irrécusables auraient pu, auraient dû enrichir le propos de Mademoiselle de Gournay. Mais une fois de plus elle se contente simplement de nom- 
mer la célèbre mère des Gracques parmi d'autres dames romaines, à peine connues et faisant l'objet chez Cicéron ou Quintilien d'une mention élogieuse, mais peu encline à lustrer la galerie des femmes savantes. Il y a Laelia (en 1634 et 1641), fille de Caïus Laelius, que Marie confond avec Sylla, malgré la mise au point de Cicéron. ${ }^{16}$ " $J$ 'ai plus d'une fois entendu causer Laelia, fille de Caüus Laelius: on la voyait toute imprégnée de l'élégance de son père," 17 rapporte l'auteur du Brutus. Quintilien, lui aussi, parle de l'élégance paternelle, ${ }^{18}$ comme si la fille avait surtout le mérite de refléter un certain talent du père. Il y a ensuite la "fille d'Hortensius," mentionnée seulement en 1641, et qui n'existe apparemment que par le nom de son père, alors que dans son Institution oratoire, Quintilien dit quand même que "le discours prononcé par Hortensia, la fille de $Q$. Hortensius, devant les triumvirs, n'est pas lu seulement pour faire honneur au sexe féminin." 19 Marie de Gournay ne rapporte pas ce mot laconique. Au lecteur curieux elle laisse le soin de découvrir que Laelia et Hortensia furent l'objet d'un "eloge celebre," expression hyperbolique s'il en est.

Plus probant sera néanmoins l'exemple d'Areté (1634 et 1641), fille et disciple d'Aristippus. C'est pour elle que Marie de Gournay s'enflamme et convainc: Areté, écrit-elle, "acquit en cette noble cité [Athènes] cent dix philosophes pour disciples, tenant publiquement la chaise que son pere avoit quittée par la mort; et comme elle eut en outre publié plusieurs excellens escrits, les Grecs l'honorerent de cet eloge: Qu'elle avoit la plume de son pere, l'ame de Socrates, la langue d'Homere."20 Voilà enfin du solide.

Il est cependant étrange que Marie de Gournay passe d'Areté, authentique femme savante, selon elle, à la reine de Saba, glorieuse, semble-t-il, parce qu'elle alla "adorer la sagesse de Salomon," et qu'une telle vénération d'un homme, selon Marie de Gournay, ne pouvait venir que d'une "correspondance de sagesse?" On sourit devant cette vague théorie de l'osmose intellectuelle, et l'on déplore que l'auteur de l'Égalité n'ait pas compris qu'un mélange hétérogène de personnages historiques très différents par le mérite et la réputation nuirait considérablement à la fameuse thèse égalitaire. Bref, la frivolité d'un exemple assombrit aisément l'éclat de celui qui le précède ou le suit.

Vient ensuite dans l'ordre, en 1634, repris en 1641, la célèbre poétesse grecque, Sappho. On ne peut contester la renommée de cette dernière, honorée qu'elle fut de toute la Grèce, même de son vivant. L'Anthologie palatine contient d'ailleurs 
plusieurs épitaphes qui lui avaient été consacrées, et dont la plus connue est sans doute celle de Platon: " $D$ 'aucuns disent qu'il y a neuf muses; mais ils se trompent, car voyez! il y a Sappho de Lesbos qui est la dixième" (IX). Dans le Phèdre de Platon, Socrate parle de la "belle Sappho" et la range parmi les sages. Deux siècles plus tard, la Rhétorique d'Aristote rapporte que "le peuple de Mitylène a honoré Sappho, bien qu'elle fût une femme." Catulle et Horace l'imitent; Plutarque la cite abondamment. Il n'y a pas de doute que Sappho a pu rivaliser avec les meilleurs poètes de son temps, comme il ressort de la qualité de son oeuvre conservée, l'Ode à Aphrodite et de quelques fragments épars. Marie de Gournay a donc eu la main heureuse en la nommant. Mais elle étaie maladroitement l'exemplarité de la grande poétesse en la mettant seulement en parallèle avec Socrate et sa méthode d'aimer, telle que rapportée par Maximus Tyrius. En effet, les subtilités de l'amour saphique sont peut-être moins susceptibles d'inspirer l'admiration, ou de prouver une supériorité intellectuelle, que ne l'étaient la sensibilité et la délicatesse musicale de la poésie amoureuse de Sappho.

D'autres femmes poètes sont honorées à partir de l'Égalité de 1634. Il s'agit de Corinne et d'Érine, cette dernière étant contemporaine de Sappho. Corinne, qui vécut un siècle après Sappho, avait remporté une victoire sur Pindare lors d'un concours de poésie. C'est ce que rapporte Pausanias dans sa Description de la Grèce: "Corinne, la seule poétesse lyrique de Tanagra, avait son tombeau en un endroit évident de la ville, et dans le gymnase se trouve une peinture de Corinne nouant un bandeau autour de sa tête, à la suite de sa victoire remportée sur Pindare à Thèbes avec un poème lyrique." 21 Quant à Érine, originaire de Telos, elle fut l'auteur d'un poème lyrique malheureusement perdu, "La Quenouille." Elle avait été l'amie de Sappho et mourut à l'âge de dix-neuf ans. L'Anthologie grecque l'a honorée dans une épitaphe, ce qui confirme sa réputation et son talent.

On ne saurait donc mettre en doute le mérite de Sappho, de Corinne ou d'Érine, même si nous ne possédons que quelques fragments de leurs oeuvres, ou que l'oeuvre de l'une ou de l'autre a été perdue. L'évocation de ces femmes poètes est peut-être une manière un peu légère de contribuer à l'idée de l'égalité des hommes et des femmes, mais le fait qu'une femme est aussi capable qu'un homme de tourner joliment quelques vers démontre quand même un équilibre à la fois spirituel et intellectuel entre les sexes. La seule chose que l'on puisse sans doute regretter, c'est que Marie de Gournay se contente d'effleurer la réputation de femmes poètes sans être en mesure de démontrer, de facto, le prestige de leurs oeuvres. Il est curieux et dommage qu'elle 
n'ait pas cru utile de parler d'une Christine de Pisan, ne fut-ce que pour son érudition, d'une Marguerite de Navarre, voire d'une Louise Labé.

Un des griefs de Marie de Gournay était que les femmes avaient été privées d'instruction, et que la discrimination dont elles avaient souffert reposait uniquement sur leur éducation déficiente. Cette analyse est juste, et c'est dans ce sens qu'il aurait fallu proposer des exemples presque contemporains, susceptibles de prouver qu'une femme instruite n'a rien à envier à un homme.

Pour honorer les femmes savantes du monde des philosophes grecs, Marie de Gournay rappelle aussi la présence de Lastémia et d'Axiothéa dans le milieu platonicien. Diogène Laërce, en énumérant les disciples de Platon, avait ajouté un détail intéressant à propos d'Axiothéa: elle "s'habillait en homme, si l'on en croit Dicéarque." 22 Reproche ou moquerie? L'intention de Diogène restera toujours impénétrable, et Marie de Gournay a préféré ignorer la remarque. Quoi qu'il en soit, c'est avec ces deux dernières figures féminines que s'épuise la nomenclature des savantes grecques.

En dépit d'une information lacuneuse au sujet de ces femmes du monde gréco-latin, l'ensemble élitiste reste suffisamment prestigieux, et supporte donc, à l'entendement d'un public très averti, la cause de l'égalité intellectuelle des hommes et des femmes.

Il est dès lors surprenant, sinon fầcheux, que Marie de Gournay ait poursuivi son argumentation en 1634 et 1641 en évoquant des femmes reconnues seulement pour leur courage et leur capacité de souffrir. Montrer "combien les Dames sont capables de cet autre triomphe de la force magnanime, qui consiste en la constance et en la souffrance des plus aspres travaux," est une entreprise louable, certes, mais qui sert très peu la cause du traité. Dans son désir de frapper fort, l'auteur ne frappe pas toujours juste. L'esprit de surenchère l'entraine, et dans un sens nuit aux desseins de la dialectique.

Comme dans une envolée oratoire, elle nomme Épicharis, Laeena, Porcia, et la mère des Machabées. La première est l'affranchie qui fut mêlée à la conspiration de Pison, et dont le courage a été rapporté dans les Annales de Tacite. Soumise à la torture par Néron, elle n'avoua rien, ce qui fit dire à Tacite: "Admirable exemple donné 
par une femme, par une affranchie qui, dans une telle extrémité, protégeait des étrangers." 23

La deuxième, Laeena (ou Leaena), fut une courtisane également soumise à la torture dans une affaire de conspiration. Elle mourut courageusement, dit Pausanias, et les Athéniens lui érigèrent une statue de bronze d'une lionne en son honneur. ${ }^{24}$ Pour ne pas répondre à ses bourreaux, elle se coupa la langue, raconte Aristote dans sa Politique.

La mort de Porcia, femme de Brutus, a été rapportée par Plutarque. ${ }^{25}$ Elle avait pris la résolution de mourir, mais lorsque ses parents voulurent l'en empêcher, elle "tira du foyer des charbons tous ardents, et les jeta dans sa bouche, qu'elle tint si étroitement fermée, qu'elle s'en étouffa."

Il est difficile d'associer tous ces actes de désespoir, et, disons-le, d'un courage quelque peu démentiel, à la thèse de l'égalité des hommes et des femmes. Nulle part dans le traité est-il question du courage des hommes, car le but avoué de Marie de Gournay n'est pas là. On pourrait donc se demander pourquoi ces exemples qui ne manquent pas d'horreur ont séduit l'imagination de l'auteur. ${ }^{26}$

L'histoire des Maccabées (Livre II) n'est pas moins horrible. Sept frères sont torturés sous les yeux de leur mère, pour avoir mangé de la viande de porc. "Quant à la mère, lit-on, elle fut tout à fait admirable et digne d'une glorieuse mémoire, elle qui, pour avoir mis.dans le Seigneur ses espoirs, supporta avec courage de voir ses sept fils mis à mort en l'espace d'un seul jour."

Pourquoi Marie de Gournay s'est-elle arrêtée à tous ces épisodes dénués de toute édification intellectuelle? Deux raisons complémentaires sont possibles. La première nous paraît d'un ordre subjectif: désireuse de dresser un inventaire persuasif de femmes remarquables, elle offre un ensemble hétérogène où s'entremêlent des savantes, des femmes courageuses et des saintes. L'égalité avec les hommes, qui soustend le traité, apparaît dès lors sous un jour strictement émotif et peu enclin à convaincre. La deuxième raison tient sans doute à l' exemple que lui avait fourni Plutarque en écrivant son De Virtute mulierum, évoqué à partir de la première version de 1622 . Mais, comme on sait, l'histoire des femmes vertueuses de Plutarque n'est point destinée à chanter les louanges du savoir féminin, mais plutôt à glorifier le courage, la fidélité, voire quelque forme subtile de soumission féminine. 
Enfin, la version de 1641, relativement fidèle à celle de 1634, allonge encore la liste des femmes célèbres du passé (ou l'alourdit-elle?) en mentionnant sainte Thècle, vierge et martyre, convertie par saint Paul, et suppliciée sans perdre courage. Vient ensuite Appia (ou Apphia), vraisemblablement la femme de Philémon à qui Paul adressa son épître. Une dernière sainte femme, Catherine de Sienne, clôt l'inventaire de Marie de Gournay qui se dit heureuse d'avoir découvert dans les écrits de François de Sales la sainte femme résistant à "la vilenie et délectation charnelle." 27 Cette ultime surenchère, car c'en est une, manque décidément de conviction, même si le combat de Catherine de Sienne contre la tentation du péché charnel suscita en elle "un grand accroissement de (sa) vertu et de (sa) force."

Il reste toutefois un dernier nom prestigieux que Marie de Gournay ajoute à la liste de 1641: celui de Mademoiselle de Schurman, ${ }^{28}$ l'illustre Hollandaise, née en 1607, femme savante et correspondante de Marie de Gournay, ainsi que des milieux humanistes de son temps. Marie l'appelle "ce nouvel astre" et elle vante et son éloquence et ses connaissances étendues en plusieurs langues. L'exemplarité de Mlle de Schurman est indéniable, mais de peu d'effet en 1641, cinq ans avant la publication de son $S$ 'il est nécessaire ou non, que les filles soient sçavantes.

Quel est donc le bilan à la suite des surenchères de 1634 et de 1641 ? Du côté des femmes savantes, ou supposées telles, nous en comptons treize. La grande majorité d'entre elles ne sont connues que par des témoignages restreints. Peu d'oeuvres à l'appui de leur savoir existent ou ont été conservées. Leur nom a survécu grâce à de fugitives reconnaissances par quelques hommes. Enfin, le nombre seul suffisait-il à imposer la thèse égalitaire?

Trois femmes poètes de l'Antiquité, dont l'incontestable Sappho, ont été ajoutées au palmarès. Mais les grandes figures féminines du patrimoine français, assurément plus convaincantes, sont restées ignorées.

Enfin, pour le courage et la piété, admirables mais peu aptes à soutenir l'égalité telle que Marie de Gournay l'envisage, un nombre considérable de noms parsèment le traité: quinze en tout, en ajoutant une allusion à la soeur de saint Grégoire.

Restent des noms de peu d'importance pour le propos: les deux Médicis, la femme de Pilate, la reine de Saba; ou des figures légendaires: Didon, Pentasilée, Camille, toutes dans Virgile. 
Ainsi, une conclusion quelque peu désabusée risque de s'imposer: c'est que la dialectique de Marie de Gournay, appuyée d'abord sur les écrits de "féministes" masculins fort suspects, s'affaiblit d'édition en édition à cause d'une surabondance de références nominales sans grand rapport avec le véritable objet du traité sur l'égalité. Au gré des années Marie de Gournay a été de plus en plus séduite par la pléthore: faiblesse rhétorique qui révèle le désir irrationnel et passionné de vaincre toutes les réticences et toutes les objections. L'Égalité des hommes et des femmes s'abattit en 1622 comme un vent de fraîcheur sur le petit monde des ergoteurs misogynes. Douze, quinze ans plus tard, alors que le triomphe des milieux féminins s'affirmait de plus en plus, le petit traité de Marie de Gournay, embarrassé dans une érudition quelque peu futile, et encombré d'un inventaire féminin sans grande consistance, donnait la pénible impression d'exhaler une cause caduque dans un dernier souffle de désespoir. 


\section{Notes}

1 Toutes nos citations sont tirées de notre édition de l'Egalité des hommes et des femmes. La page apparait entre parenthèses.

2 Pour Didon, Enéide, I, 653-54; pour Pentasilée, Enéide, I, 490-91.

${ }^{3}$ Pour Olda, II, Rois, XXII, 14-20 et II Chroniques, XXXIV, 22-28; pour Debora, Les Juges, IV, 4 et ss., et V, 1 et ss. Voir aussi Luc, II, 17 et XXI, 9, et Jérôme, Lettres, III, p.141.

${ }^{4}$ Voir Plutarque, Les Vies des hommes illustres, I, Périclès.

5 Voir Autores Historiae Ecclesiasticae (1539), dans Tripartitae Liber XI. Voir aussi Gilles Ménage, Historia Mulierum Philosopharum (1690).

6 Noémi Hepp, "A la recherche du 'mérite des dames," dans Destins et enjeux du XVIIe siècle, p.113.

7 Nous renvoyons à notre édition critique.

8 Sur les relations de Jérôme avec les saintes femmes, fondatrices des premiers monastères, voir le Dictionnaire d'Archéologie chrétienne et de Liturgie, VII, 2e partie.

${ }^{9}$ La fille d'alliance de Montaigne, Marie de Gournay.

10 Diogène Laërce, Vie, doctrines et sentences des philosophes illustres, VIII, Pythagore, 8 et 21.

11 Op. cit.

12 Tous ces détails sont dans Gilles Ménage, Op. cit. 
13 Brutus, 104 et 211.

14 Institution oratoire, I, 1, 6.

15 Vie de Tiberius et de Caïus Gracchus.

16 De Oratore, III, 45.

17 Brutus, 211.

18 Instituition oratoire, I, 1, 6.

${ }^{19}$ Institution oratoire, I,1,6.

20 Diogène Laërce dit qu'Aristippus “éleva sa fille dans d'excellents principes, l'exerçant à mépriser le superflu"(II, Aristippus, 72).

${ }^{21}$ La Boétie, XXII, 3.

22 Platon, III, 46.

23 Annales, XV, 52.

${ }^{24}$ Description de la Grèce, Attica, XXIII, 2.

${ }^{25}$ Marcus Brutus.

26 Il est vrai que le goût de l'horreur n'a jamais rebuté Marie de Gournay. Son Proumenoir de Monsieur de Montaigne contient quelques scènes de choix. Plusieurs exemples dans l'Egalité, on l'aura remarqué, tournent autour de la torture!

27 François de Sales, Introduction à la vie dévote, ch. IV.

28 Mario Schiff lui consacre une belle notice. 


\section{Bibliographie}

Amyot, Jacques. Plutarque. La vie des hommes illustres. Ed. G. Walter. Paris: Gallimard, La Pléiade, 1951.

Amyot, Jacques. Les Oeuvres morales de Plutarque. Lyon: Estienne Michel, 1588.

Aristote. Politique. Paris: Les belles lettres, 1986-91.

Aristote. Rhétorique. Paris: Les belles lettres, 1989-91.

Anthologie grecque. Anthologie palatine. Paris: Les belles lettres, 1960-1970.

Autores historiae ecclesiasticae. Basileae: anno 1539.

Cicéron. Brutus. Ed. J. Martha. Paris: Les belles lettres, 1960.

Dictionnaire d'Archéologie chrétienne et de Liturgie. Paris: Letouzey et Ane, 1907.

Diogène Laërce. Vie, doctrines et sentences des philosophes illustres. Ed. R. Grenaille. Paris: Garnier-Flammarion, 1965.

Gournay, Marie de. Égalité des hommes et des femmes, Grief des dames, suivis du Proumenoir de Monsieur de Montaigne. Ed. critique par Constant Venesoen. Genève: DROZ, 1993.

Hepp, Noémie. A la recherche du "mérite des dames," dans Destins et enjeux du XVITe siècle. Paris: PUF, 1985.

Jérôme (saint). Lettres. Paris: Les belles lettres, 1951-58.

La Bible, 3 vol. Paris: Gallimard, la Pléiade, 1956-71.

Ménage, Gilles. Historia mulierum philosopharum. Lugduni: Claudium Rigaud, 1690. Mora, E. Sappho, histoire d'un poète. Paris: Flammarion, 1966. 
Mora, E. Sappho, histoire d'un poète. Paris: Flammarion, 1966.

Pausanias. The description of Greece. London: Heinemann, 1918.

Platon. Oeuvres complètes, I \& II. Paris: Gallimard, la Pléiade, 1950.

Quintilien. Institution oratoire. Paris: Les belles lettres, 1975.

Sales, Francois de. Oeuvres. Introduction à la vie dévote. Paris: Gallimard, la Pléiade, 1969.

Schiff, Mario. La fille d'alliance de Montaigne, Marie de Gournay. Paris: $\mathrm{H}$. Champion, 1910.

Tacite. Annales. Paris: Les belles lettres, 1958-62.

Virgile. Énéide. Paris: Les belles lettres, 1977-80.

Wright, F.A. The Poets of the Greek Anthology. New York: Routledge and sons, 1924. 